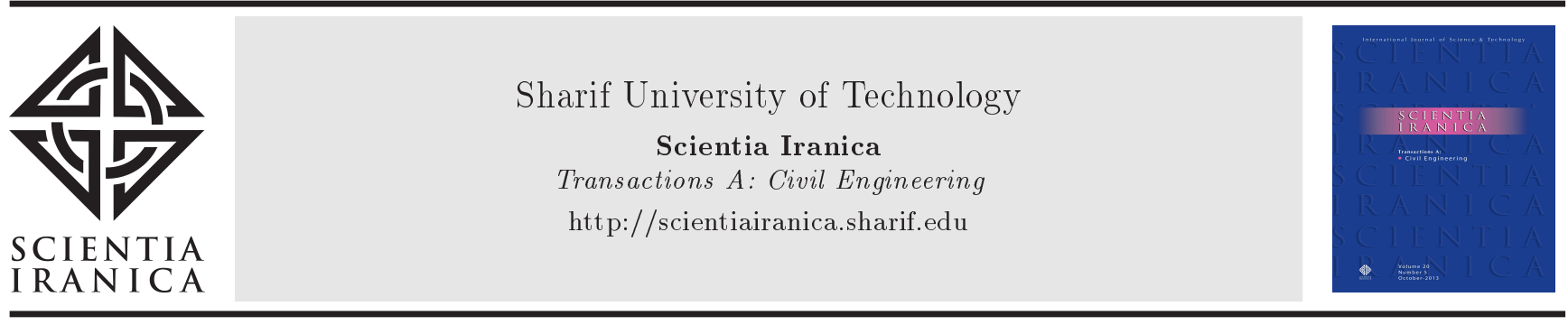

\title{
Mean bed shear stress estimation in a rough rectangular channel using a hybrid genetic algorithm based on an artificial neural network and genetic programming
}

\author{
Z. Sheikh Khozani, H. Bonakdari*, and A.H. Zaji \\ Department of Civil Engineering, Razi University, Kermanshah, Iran. \\ Received 23 February 2016; received in revised form 16 May 2016; accepted 19 September 2016
}

\author{
KEYWORDS \\ Artificial neural \\ network; \\ Bed shear stress; \\ Genetic algorithm; \\ Genetic programming; \\ Hybrid soft computing \\ models; \\ Rough rectangular \\ channels.
}

\begin{abstract}
The determination of erosion and deposition patterns in channels requires detailed knowledge and estimation of the bed shear stress. In this investigation, the application of a Genetic Algorithm-based Artificial (GAA) neural network and Genetic Programming (GP) was presented to predict bed shear stress in a rectangular channel with rough boundaries. Several input combinations, fitness functions, and transfer functions were investigated to determine the best GAA model. Also, the effect of various GP operators on estimating bed shear stress was studied. A comparison between the GAA and GP techniques' abilities to predict bed shear stress was made and then investigated. The results revealed that the GAA model performs better in predicting the bed shear stress $($ RMSE $=0.0774)$, as compared to the GP model $(\mathrm{RMSE}=0.0835)$.

(C) 2018 Sharif University of Technology. All rights reserved.
\end{abstract}

\section{Introduction}

The flow structure in open channels is directly affected by the shear stress distribution along the wetted perimeter; hence, the variations of the boundary shear stress are more important. It has long been established that many factors influence this distribution. These factors include variation of the longitudinal and lateral boundary roughness types, geometry of a cross-section, longitudinal variation of plan-form geometry, and sediment concentration and deposition in the bed.

Many direct and indirect methods have been used to calculate the shear stress along a wetted

\footnotetext{
*. Corresponding author. Tel.: +98 $831427453 \%$;

Fax: +988314283264

E-mail addresses: y.sheikh2131@gmail.com (Z. Sheikh

Khozani); bonakdari@yahoo.com (H. Bonakdari);

amirzaji@gmail.com (A.H.Zaji)
}

doi: $10.24200 /$ sci.2017.4256 perimeter [1-8]. Guo and Julien [9] and BabaeyanKoopaei et al. [10] found that the open channel flow for the separation of bed and wall shear stress was more important for a velocity profile study, while Julien [11] and Berlamont et al. [12] found that it was more important for the bed load transport estimation.

In addition to experimental studies, some researchers have focused on determining shear stress using analytical models based on continuity and momentum equations $[13,14]$. Using the entropy concept, the shear stress distribution was studied along the wall and bed of a channel $[15,16]$. Berlamont et al. [12] used computational fluid dynamic modeling to estimate the shear stress distribution in a rectangular channel and partially filled pipes.

Recently, the application of soft computing techniques has been widely used for the prediction of hydraulic phenomena [17-19]. Najafzadeh et al. [20] applied Group Method of Data Handling (GMDH) network to predict abutments scour depth of bridges. 
Some researchers utilized GEP model to solve different hydraulic problems [21-23]. Azamathulla and Zahiri [24] used linear genetic programming to predict the flow discharge in a compound open channel. Zaji and Bonakdari [25] utilized the ANN and Radial Basis Neural Network (RBNN) to compute the discharge capacity of a triangular side weir. The suspended sediment load was estimated using soft computing techniques by Tayfur [26] and Kisi et al. [27]. Cobaner et al. [28] used a simple ANN method to estimate percentage of shear force carried by walls in rectangular ducts and channels. Sheikh Khozani et al. [29], by applying GP and GAA models, could predict percentage of shear force carried by walls $\left(\% S F_{w}\right)$ of rough rectangular open channels. The authors expressed that the ANN model has better performance in predicting shear force than the traditional shear force relationships. Predicting $\% S F_{w}$ in smooth rectangular channels was studied by Sheikh Khozani et al. [30] using GP and GAA methods.

Because the bed shear stress has an important role in bed load sediment transition, a few studies have been conducted on the rectangular channel with rough boundaries using the soft computing technique. Consequently, the aim of this study is to utilize this technique in estimating the bed shear stress in a rectangular channel with non-homogenous roughness. For this goal, the GAA method as a novel combination of modified Genetic Algorithm (GA) and Artificial Neural Network (ANN) was used to predict the bed shear stress. The performance of the GAA was compared with that of the Genetic Programming (GP) method, as a practical branch of the GA. Also, the obtained equation by the best method has been compared with the equation of Knight [2].

\section{Materials and methods}

\subsection{Genetic algorithm-based artificial neural network}

The multi-layer perceptron ANN is widely used in various engineering fields as a successful soft computing method. An ANN method is composed of three major layers: one input layer, one or more hidden layers, and one output layer. Each layer consists of some neurons. The input layer's neurons are the input variables of the considered problem. In the current ANN model, the output layer has one neuron, i.e., the output of the problem. Each hidden layer has some neurons. Each neuron in the hidden layers, at first, compute the weighted sums of neurons in last layer, and then by putting the results of the summation in the activation functions, the estimation for each neuron is calculated.

The activation function selection significantly affects the performance of the model [31-33]. In this study, four combinations of activation functions were examined to determine the most appropriate one of all. The considered activation functions include the logarithmic sigmoid (Eq. (1)), hyperbolic tangent sigmoid (Eq. (2)), and linear (Eq. (3)):

$$
\begin{aligned}
& \log \operatorname{sig}(x)=\frac{1}{1+e^{-x}}, \\
& \operatorname{tansig}(x)=\frac{2}{1+e^{-2 x}}-1, \\
& \operatorname{purelin}(x)=x
\end{aligned}
$$

In addition to the advantages of the ANN method in modeling the complex problems, the absence of a specific rule in choosing the number of hidden layer nodes makes the modeling process more puzzling. In this study, a modified GA was used to optimize the structure of the ANN method. The GA investigates different ANN models with various numbers of neurons in hidden layers and automatically chooses the most appropriate one.

Levenberg-Marquardt Algorithm [34] was used to train the ANN model. It is possible that a good combination of a number of hidden layer neurons was eliminated by the GA due to bad luck with the Levenberg-Marquardt training process and random nature of the training algorithms used. So, the GA used to optimize the ANN model needs some modifications.

The modified GA used in the optimization of the ANN structure is shown in Figure 1. According to Figure 1, the GA modification was conducted in the elite populations. The elite population consists of the best $15 \%$ of the entire chromosomes. Each individual in the elite population was run with the ANN model several times. The best cost for each individual was replaced in the elite population.

This modification has two major benefits. First, it prevents the local minimum trapping of the GA. Second, it minimizes the effect of the random nature of the Levenberg-Marquardt training process. Finally, the GAA method, as the combination of the modified GA and ANN methods, was introduced.

\subsection{Genetic programming}

The GP method, as a branch of the GA algorithm, was developed by Koza [35]. The general processing rules of the GP method are similar to those of the GA algorithm. The rules are as follows. The initial population was performed by a number of chromosomes. Here, the chromosomes are the programs. The error of each chromosome was investigated by the deified fitness function.

The aim of GP processing is to find the most appropriate program that could be used to model the considered problem. Because of this, the GP method explicitly becomes a suitable method for the 


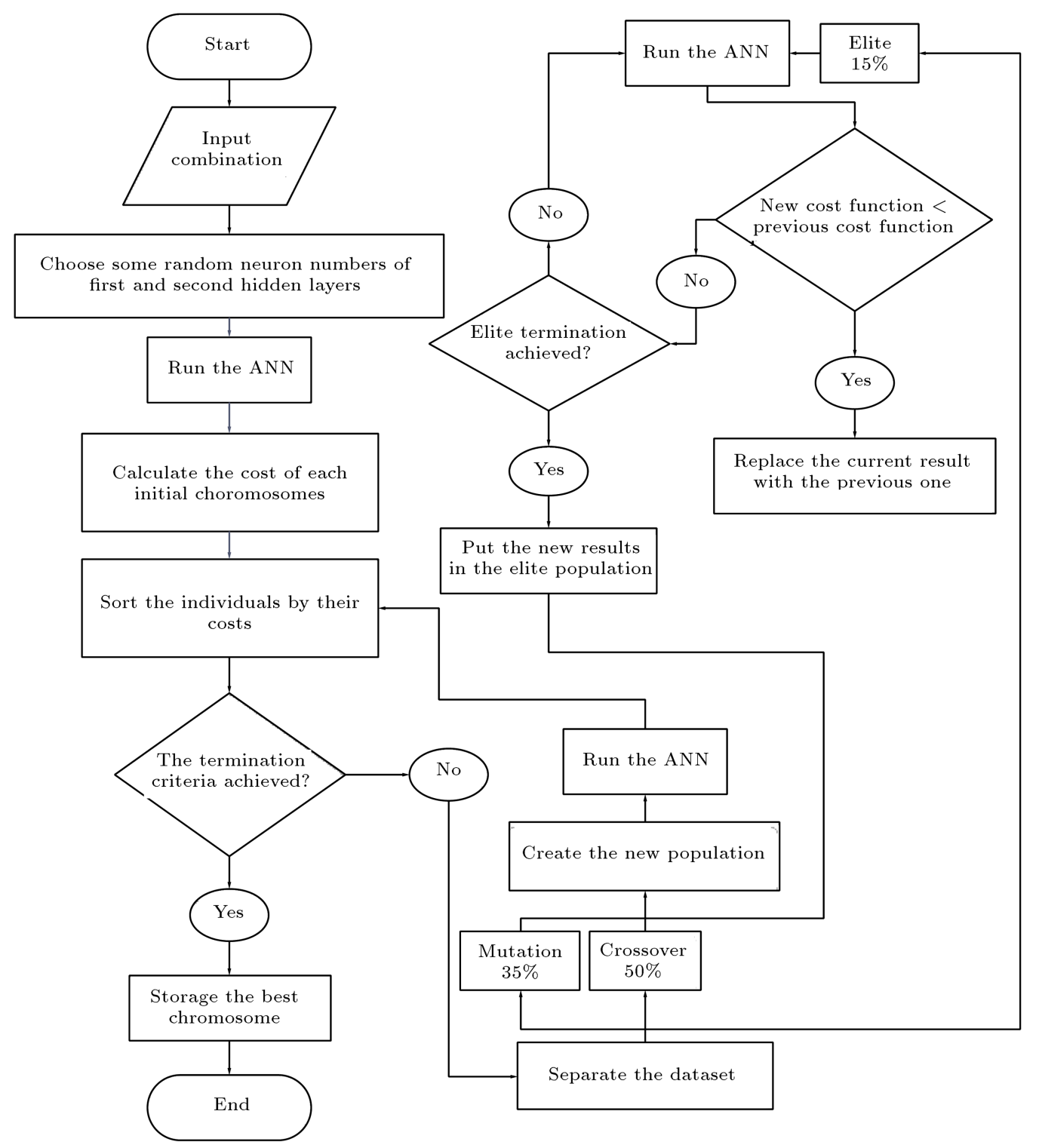

Figure 1. Flowchart of the GAA model

practical situations. To find an appropriate GP model in each problem, some of the properties should be specified.

Each GP program uses some functions that must be determined. The functions include arithmetic operations (e.g.,,,$+- \times$, and $\div$ ), mathematical functions (e.g., sin, cos, and power), and logical functions (e.g., AND, OR, and NOT). There is no specific rule in function determination, and it should be determined for each problem. In this study, to find the most appropriate functions, four different function combinations were examined.

The processing steps of the GP are as follows: (1) Selecting the initial population of the individuals (programs) randomly; (2) Calculating the cost of each individual by the fitness function; (3) Sorting the individuals according to the calculated costs; (4)
Conducting the reproduction, mutation, and crossover on the population and performing the new population; (5) Achieving one of the termination criteria (e.g., the time of the run, number of generations, and value of the error) in the process. Table 1 shows the characteristics of the GP model used.

\subsection{Case study}

The dimensionless mean bed shear stress data of the experiments were used; these were originally conducted by Knight [2]. By using the Preston pipe technique, Knight [2] calculated the bed shear stress along a flume that was $15 \mathrm{~m}$ in length, $460 \mathrm{~mm}$ in width, and with a constant bed slope of $9.58 \times 10^{-4}$. This flume had a rectangular cross-section with rough boundaries and shear stress that was measured at different flow depths. The ranges of the geometric and hydraulic characteris- 
Table 1. The characteristics of the GP model.

\begin{tabular}{cc}
\hline $\begin{array}{c}\text { Parameter } \\
\text { name }\end{array}$ & $\begin{array}{c}\text { Parameter } \\
\text { specification }\end{array}$ \\
\hline Population size & 500 \\
Mutation frequency & $93 \%$ \\
Crossover frequency & $50 \%$ \\
Number of replication & 10 \\
Block mutation rate & $30 \%$ \\
Instruction mutation rate & $30 \%$ \\
Instruction data mutation rate & $40 \%$ \\
Homologous crossover & $95 \%$ \\
Program initial size & 64 \\
Program maximum size & 256 \\
Function combination & To be determined \\
Fitness function & To be determined \\
\hline
\end{tabular}

tics of the rectangular channel used by Knight [2] are listed in Table 2.

Knight [2] presented an empirical equation for calculating the mean bed shear stress as follows:

$$
\frac{\bar{\tau}_{b}}{\rho g h S_{f}}=1-0.01\left(\% S F_{w}\right),
$$

where $\bar{\tau}_{b}$ is the mean bed shear stress, $S_{f}$ is the energy slope, $h$ is the water depth, $\rho$ is the fluid density, and $\% S F_{w}$ is the total shear force carried by the walls. This total shear force can be calculated as follows:

$$
\% S F_{\mathrm{w}}=e^{\alpha}\left(\tanh (\pi \beta)-0.5[\tanh (\pi \beta)-\beta]^{2}\right)
$$

where $\alpha=-3.264 \log \left(\frac{B}{h}+3\right)+6.211, \beta=1-\frac{\gamma}{5}$, and $\gamma=\log \left(\frac{k_{s b}}{k_{s w}}\right)$.

The shear stress depends on the hydraulics of the flow, cross-section, and bed and wall roughness. The dependent parameters are: flow velocity $(V)$, hydraulic radius $(R)$, fluid density $(\rho)$, geometry of the channel $(B)$, bed and wall roughness $\left(k_{s b}, k_{s w}\right)$, flow depth $(h)$, and energy slope $\left(S_{f}\right)$. A functional relationship between the bed shear stress and the effective parameters can be expressed as follows:

$$
\bar{\tau}_{b}=F\left(\rho, g, B, h, k_{s b}, k_{s w}, R, S_{f}, V\right) .
$$

Using a dimensional analysis for expressing the dimensionless bed shear stress, the following function was obtained:

$$
\frac{\bar{\tau}_{b}}{\rho g h S_{f}}=f\left(\frac{B}{h}, \frac{k_{s b}}{k_{s w}}, \text { Fr, Re }\right),
$$

where $\frac{B}{h}$ is the aspect ratio, $\frac{k_{s b}}{k_{s w}}$ is the relative roughness, Fr is the Froude number, and Re is the Reynolds number.

Table 2 shows the ranges of data set parameters that were used in modeling. About $75 \%$ of all data were selected randomly for training, and the remaining data were used for testing stage.

Eq. (4) was also selected to predict the mean bed shear stress and was compared with the proposed model.

\section{Application and results}

In order to investigate the accuracy of the models and the fitness function, six statistical parameters were used. These parameters include: the Root Mean Squared Error (RMSE), Mean Squared Error (MSE), Mean Absolute Error (MAE), average absolute deviation $(\delta \%)$, Scatter Index (SI), and BIAS as follows:

$$
\begin{aligned}
& \mathrm{RMSE}=\sqrt{\frac{\sum_{i=1}^{n}\left(\bar{\tau}_{i p}-\bar{\tau}_{i m}\right)^{2}}{n}}, \\
& \mathrm{MSE}=\frac{\sum_{i=1}^{n}\left(\bar{\tau}_{i p}-\bar{\tau}_{i m}\right)^{2}}{n}, \\
& \mathrm{MAE}=\frac{1}{n} \sum_{i=1}^{n}\left|\bar{\tau}_{i p}-\bar{\tau}_{i m}\right|, \\
& \delta=\left(\frac{\sum_{i=1}^{n}\left|\bar{\tau}_{i p}-\bar{\tau}_{i m}\right|}{\left.\sum_{i=1}^{n} \bar{\tau}_{i p}\right) \times 100,}\right. \\
& \mathrm{SI}=\left(\sqrt{\frac{\sum_{i=1}^{n}\left(\bar{\tau}_{i p}-\bar{\tau}_{i m}\right)^{2}}{n}}\right)\left(\frac{n}{\sum_{i=1}^{n} \bar{\tau}_{i m}}\right),
\end{aligned}
$$

Table 2. Ranges of the geometric and hydraulic variables of the rectangular channel.

\begin{tabular}{cccc}
\hline Symbol & Variable definition & Variable range & Mean value \\
\hline$h(\mathrm{~m})$ & Flow depth & $0.0307-0.3107$ & 0.1413 \\
$\frac{k_{s b}}{k_{s w}}$ & Relative roughness & $1-17120$ & 4255.14 \\
$Q\left(\mathrm{~m}^{3} / \mathrm{s}\right)$ & Discharge & $0.003-0.1136$ & 0.0336 \\
$V(\mathrm{~m} / \mathrm{s})$ & Velocity & $0.161-0.805$ & 0.4187 \\
$\frac{\bar{\tau}_{b}}{\rho g h S}$ & Dimensionless bed shear stress & $0.364-1.0255$ & 0.751 \\
\hline
\end{tabular}




$$
\mathrm{BIAS}=\frac{\sum_{i=1}^{n} \bar{\tau}_{i p}-\bar{\tau}_{i m}}{n},
$$

where $\bar{\tau}_{i p}$ shows the value of the predicted mean bed shear stress by the model, and $\bar{\tau}_{i m}$ is the measured mean bed shear stress in the laboratory.

To compute the mean bed shear stress, eight different input combinations were tested. These input combinations were:

(i) $B / h$, Fr and $k_{s b} / k_{s w}$;

(ii) $B / h$, Fr and Re;

(iii) $B / h$, Fr and $k_{s b} / k_{s w}$;

(iv) $B / h$, Re and $k_{s b} / k_{s w}$;

(v) $\mathrm{Fr}, k_{s b} / k_{s w}$ and Re;

(vi) $B / h$ and $k_{s b} / k_{s w}$;

(vii) $B / h$ and Fr;

(viii) $\operatorname{Fr}$ and $k_{s b} / k_{s w}$.

The results were compared with the RMSE, MAE, and $\% \delta$ statistic parameters.

In the first step of the GAA model, by assuming MSE as a fitness function and the logarithmic transfer function, the mentioned input combinations were investigated. As shown in Table 3, the GAA model with the input combination of (ii) was more accurate with the smallest values of the statistical parameters. Various GP models were developed using the same input combinations as the GAA model. Similar to the GAA model in the GP model, the assumption fitness function in the first step was that the MSE and default function $(+,-, \times, \div)$ were used to select the best input combination. As shown in Table 4, the input combination (i) is more appropriate than the others with RMSE of 0.0835 .

Continuing to use the selected input combination, a comparison was checked between the MSE and MAE to find a more appropriate fitness function in the GAA model. The results of this comparison are presented in Table 5. According to the results, it is clear that the MSE fitness function provides more accurate results with RMSE of 0.0774 than the MAE fitness function with RMSE of 0.0918. To select the best fitness function in the GA model, MSE and MAE were investigated. From Table 5, it is clear that the MSE fitness function (based on the absolute error) produces the best results for computing the mean bed shear stress. Consequently, the MSE fitness function with better statistical results $(\mathrm{RMSE}=0.0835, \mathrm{MAE}=$ 0.0732 , and $\delta \%=10.1635)$ was selected.

In the final step, four different conditions were studied by the GAA model using the logarithmic, purelin and hyperbolic tangent, as the transfer functions. The outcomes (Table 6) show that the GAA model with the logarithmic transfer function in a hidden layer and the purelin transfer function in the output layer operates better between the transfer functions.

The selection of the basic operators in building

Table 3. Statistical measures of the models in the test period of GAA model.

\begin{tabular}{llccccc}
\hline \multirow{2}{*}{ Model inputs } & \multicolumn{5}{c}{ GAA } \\
\cline { 3 - 6 } & & RMSE & MAE & $\% \boldsymbol{\delta}$ & SI & BIAS \\
\hline (i) & $B / h$, Fr and $k_{s b} / k_{s w}$ & 0.0875 & 0.0711 & 9.7614 & 0.1239 & 0.0228 \\
(ii) & $B / h$, Fr and Re & 0.0774 & 0.0631 & 8.6560 & 0.1096 & 0.0229 \\
(iii) & $B / h$, Fr and $k_{s b} / k_{s w}$ & 0.0942 & 0.0755 & 10.5406 & 0.1335 & 0.0106 \\
(iv) & $B / h$, Re and $k_{s b} / k_{s w}$ & 0.0836 & 0.0618 & 8.7320 & 0.1184 & 0.0019 \\
(v) & Fr, $k_{s b} / k_{s w}$ and Re & 0.0811 & 0.0672 & 9.0700 & 0.1149 & 0.0352 \\
(vi) & $B / h$ and $k_{s b} / k_{s w}$ & 0.0910 & 0.0738 & 10.2469 & 0.1289 & 0.0143 \\
(vii) & $B / h$ and Fr & 0.0844 & 0.0718 & 9.9990 & 0.1253 & 0.0123 \\
(viii) & Fr and $k_{s b} / k_{s w}$ & 0.1099 & 0.0893 & 11.9109 & 0.1557 & 0.0434 \\
\hline
\end{tabular}

Table 4. Statistical measures of the models in the test period of GP model.

\begin{tabular}{llccccc}
\hline \multirow{2}{*}{ Model inputs } & \multicolumn{5}{c}{ GAA } \\
\cline { 3 - 7 } & & RMSE & MAE & $\% \boldsymbol{\delta}$ & SI & BIAS \\
\hline (i) & $B / h$, Fr and $k_{s b} / k_{s w}$ & 0.0835 & 0.0732 & 10.1635 & 0.1183 & 0.0139 \\
(ii) & $B / h$, Fr and Re & 0.0909 & 0.0757 & 10.3231 & 0.1287 & 0.0272 \\
(iii) & $B / h$, Fr and $k_{s b} / k_{s w}$ & 1.6866 & 0.5342 & 47.6534 & 2.389 & 0.4151 \\
(iv) & $B / h$, Re and $k_{s b} / k_{s w}$ & 0.0919 & 0.0743 & 10.2097 & 0.1302 & 0.0213 \\
(v) & Fr, $k_{s b} / k_{s w}$ and Re & 0.0957 & 0.0821 & 11.2094 & 0.1356 & 0.0267 \\
(vi) & $B / h$ and $k_{s b} / k_{s w}$ & 0.0978 & 0.0831 & 11.0748 & 0.1385 & 0.0440 \\
(vii) & $B / h$ and Fr & 0.0879 & 0.0785 & 10.8536 & 0.1245 & 0.0169 \\
(viii) & Fr and $k_{s b} / k_{s w}$ & 0.1181 & 0.0993 & 12.8157 & 0.1860 & 0.0730 \\
\hline
\end{tabular}


Table 5. Fitness function selection for the models.

\begin{tabular}{cccc}
\hline Models & $\begin{array}{c}\text { Statistical } \\
\text { parameters }\end{array}$ & MSE & MAE \\
\hline \multirow{4}{*}{ GAA } & RMSE & 0.0774 & 0.0918 \\
& MAE & 0.0631 & 0.0734 \\
& SI $\delta$ & 8.6560 & 10.4547 \\
& BIAS & 0.1096 & 0.1300 \\
GP & & & \\
& RMSE & 0.0835 & 0.0957 \\
& MAE & 0.0732 & 0.0813 \\
& SI & 10.1635 & 11.0186 \\
& BIAS & 0.1183 & 0.1356 \\
\hline
\end{tabular}

the parse tree is the final step in the GA model. The basic range of the investigated functions is:

$$
\begin{aligned}
& F 1=\{+,-, \times, \div\} \\
& F 2=\{+,-, \times, \div, \sin (x), \cos (x)\} \\
& F 3=\{+,-, \times, \div, \sin (x), \cos (x),|x|, \sqrt{ }, \text { Power }\} .
\end{aligned}
$$

A set of preliminary model runs was carried out to test the performance of the models with the function sets and select the best one to use in the next stage of the study. All of these procedures were conducted for input combination (i) by the MSE fitness function. The investigated results of these sets of functions are illustrated in Table 7. Based on the comparison of the various GP operators listed in this table, it can be deduced that the GP default operator function set $(F 1)$ surpasses all of the other structures. It was determined that the simple operator function is better to use than the other function sets, since the other operator function sets resulted in complicated computations.

Finally, the comparison between the GAA and GP models was conducted. As can be seen in Table 8, the GAA model with an RMSE of 0.0774 was compared to the GP model with an RMSE of 0.0835. The GA model was selected due to its better performance. The GP model is capable of predicting the mean bed shear stress, too. When comparing the GAA predictions with the measured data for the test stage, the proposed model was proved to be highly capable to estimate the mean bed shear stress with few errors.

With regard to selecting the GAA model as the best model, the equation obtained from the proposed model with input combination (i), MSE fitness function, and logarithmic and linear transfer functions in the hidden and output layers, respectively, are as follows:

$$
\begin{aligned}
& \frac{\bar{\tau}_{b}}{\rho g h S}=\operatorname{purelin}\left(\left(\operatorname { l o g s i g } \left(\left(\operatorname{logsig}\left(\text { input } \times i w+b_{1}\right)\right)\right.\right.\right. \\
& \left.\times\left(w+b_{2}\right)\right) \times o w+b_{3}, \\
& \text { input }=\left[\begin{array}{lll}
\frac{B}{h} & \operatorname{Fr} & \operatorname{Re}
\end{array}\right] \\
& i w= \\
& {\left[\begin{array}{cccccc}
0.451 & -0.122 & 0.348 & -0.349 & -0.225 & -0.006 \\
-26.623 & -20.878 & 21.350 & 24.510 & 25.914 & 21.037 \\
0.000 & -0.001 & 0.000 & 0.000 & 0.000 & 0.000
\end{array}\right](15)} \\
& l w=\left[\begin{array}{cccc}
-3.167 & 1.971 & 0.045 & 2.700 \\
2.451 & -4.757 & 5.707 & -3.610 \\
-0.715 & 0.687 & 1.799 & -3.610 \\
4.661 & -2.240 & -3.713 & 3.809 \\
2.605 & 1.191 & -0.280 & 1.207 \\
-2.093 & 4.041 & -0.244 & -0.435
\end{array}\right]
\end{aligned}
$$

Table 6. Transfer function selection process for the GAA model.

\begin{tabular}{ccccccc}
\hline $\begin{array}{c}\text { Hidden layers } \\
\text { transfer function }\end{array}$ & $\begin{array}{c}\text { Output layer } \\
\text { transfer function }\end{array}$ & RMSE & MAE & $\% \boldsymbol{\delta}$ & SI & BIAS \\
\hline logsig & purelin & 0.0774 & 0.0631 & 8.6560 & 0.1096 & 0.0229 \\
logsig & logsig & 0.0833 & 0.0719 & 9.9282 & 0.1180 & 0.0179 \\
tansig & purelin & 0.0952 & 0.0790 & 10.8712 & 0.1348 & 0.0206 \\
tansig & tansig & 0.0817 & 0.0658 & 8.9494 & 0.1158 & 0.0289 \\
\hline
\end{tabular}

Table 7. Statistical parameters for the preliminary selection of the basic functions for the parse tree.

\begin{tabular}{ccccccc}
\hline & Definition & RMSE & MAE & $\% \boldsymbol{\delta}$ & SI & BIAS \\
\hline$F 1$ & $\{+,-, \times, \div\}$ & 0.0835 & 0.0732 & 10.1635 & 0.1183 & 0.0139 \\
$F 2$ & $\{+,-, \times, \div, \sin (x), \cos (x)\}$ & 0.1048 & 0.0926 & 12.5872 & 0.1484 & 0.0296 \\
$F 3$ & $\{+,-, \times, \div, \sin (x), \cos (x),|x|, \sqrt{ }$, Power $\}$ & 0.0988 & 0.0772 & 10.9509 & 0.1400 & -0.0011 \\
\hline
\end{tabular}


Table 8. Comparison between the GAA and GP models.

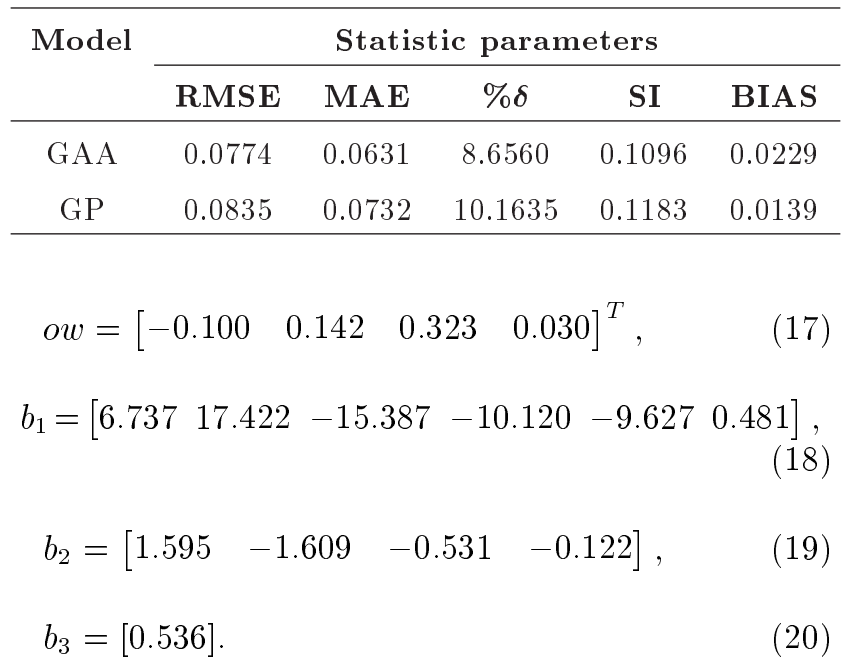

In Figure 2, the calculated mean bed shear stress of the GAA and GP models for the training step were presented in the form of a scatterplot. As can be seen, the GAA model predicts the mean bed shear stress better than the GP model does for training processes. The GAA model estimates were found to be closer to the corresponding observed values than those of the GP model were. As seen, the two models' predictions underestimated shear stress values for some cases and overestimated for other cases. Figure 3 illustrates the estimates of the GAA and GP models in the form of a scatterplot for test dataset. It can be clearly seen from the scatterplots that the estimates of the GAA model are less scattered and closer to the exact line than those of the GP model. According to the results, performance of the GAA model in estimating bed shear stress is higher than that of the GP model. Therefore, the GAA model was selected as the best model to estimate bed shear stress in rectangular channels with flat bed.

Table 9 shows the comparison between the obtained equation of the best model (GAA) and the presented equation by Knight [2]. The results show that the GAA model has a lower error of mean bed shear stress $(\mathrm{RMSE}=0.0731, \mathrm{MAE}=0.0607$, and $\% \delta=8.0451$ ) for a rectangular channel with rough boundaries than that of the equation of Knight [2] (Figure 4). According to these results, the GAA model is much closer to the exact line than the equation of Knight [2], and hence, is more accurate. Therefore,
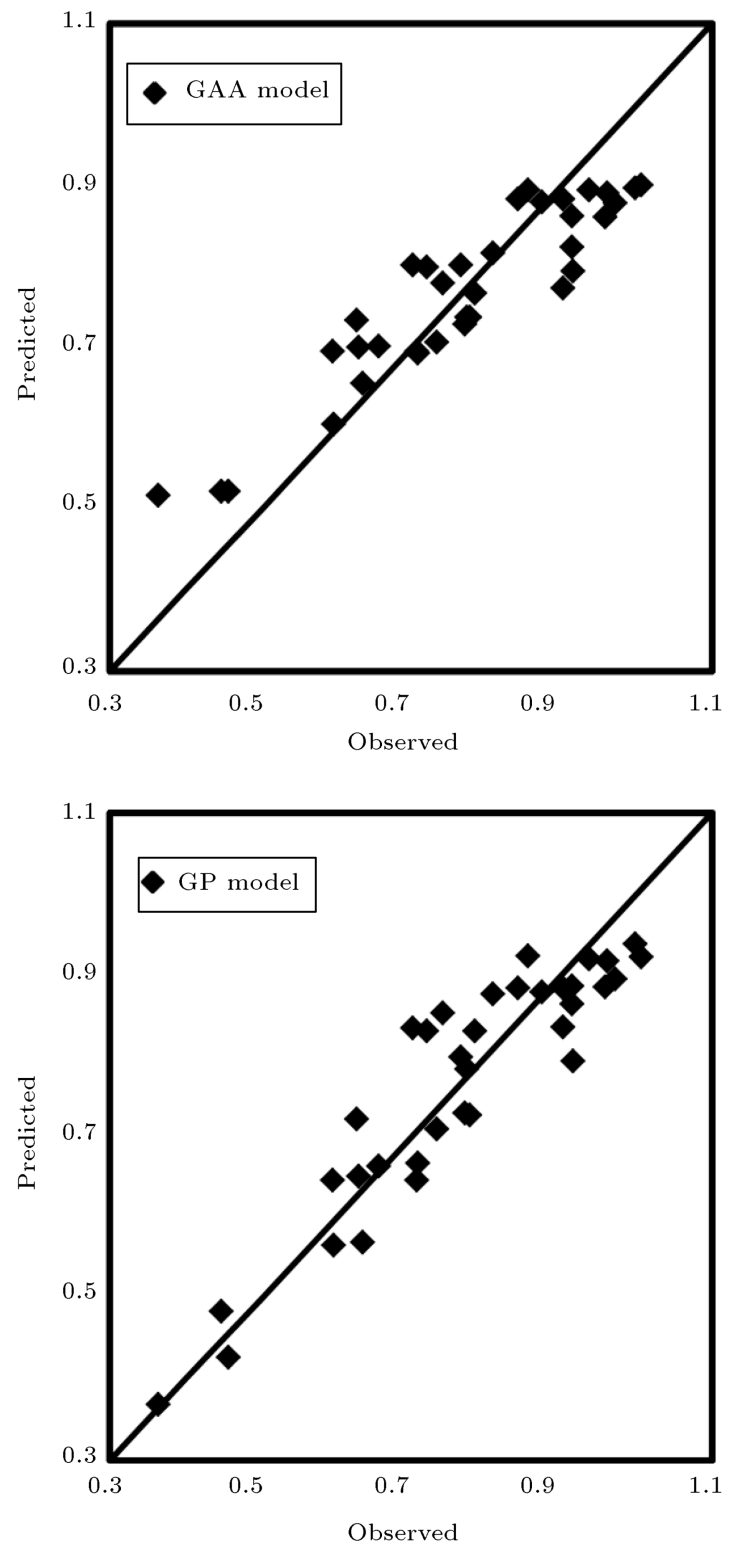

Figure 2. Observed versus computed mean bed shear stress of the GAA and GP models for a training test.

the obtained equation by the GAA model is more applicable in rectangular channel with rough boundaries and can be used instead of the equation proposed by Knight [2].

In the proposed equation, the Froude and Reynolds numbers are effective in calculating the mean bed shear stress, in addition to the aspect ratio;

Table 9. Comparison between the GAA models and the equation of Knight [2].

\begin{tabular}{cccccc}
\hline \multirow{2}{*}{ Model } & \multicolumn{5}{c}{ Statistic parameters } \\
\cline { 2 - 6 } & RMSE & MAE & $\boldsymbol{\%} \boldsymbol{\delta}$ & SI & BIAS \\
\hline GAA Model & 0.0731 & 0.0607 & 8.0451 & 0.0974 & 0.0046 \\
Equation of Knight [2] & 0.0804 & 0.0683 & 9.0925 & 0.1044 & -0.0192 \\
\hline
\end{tabular}



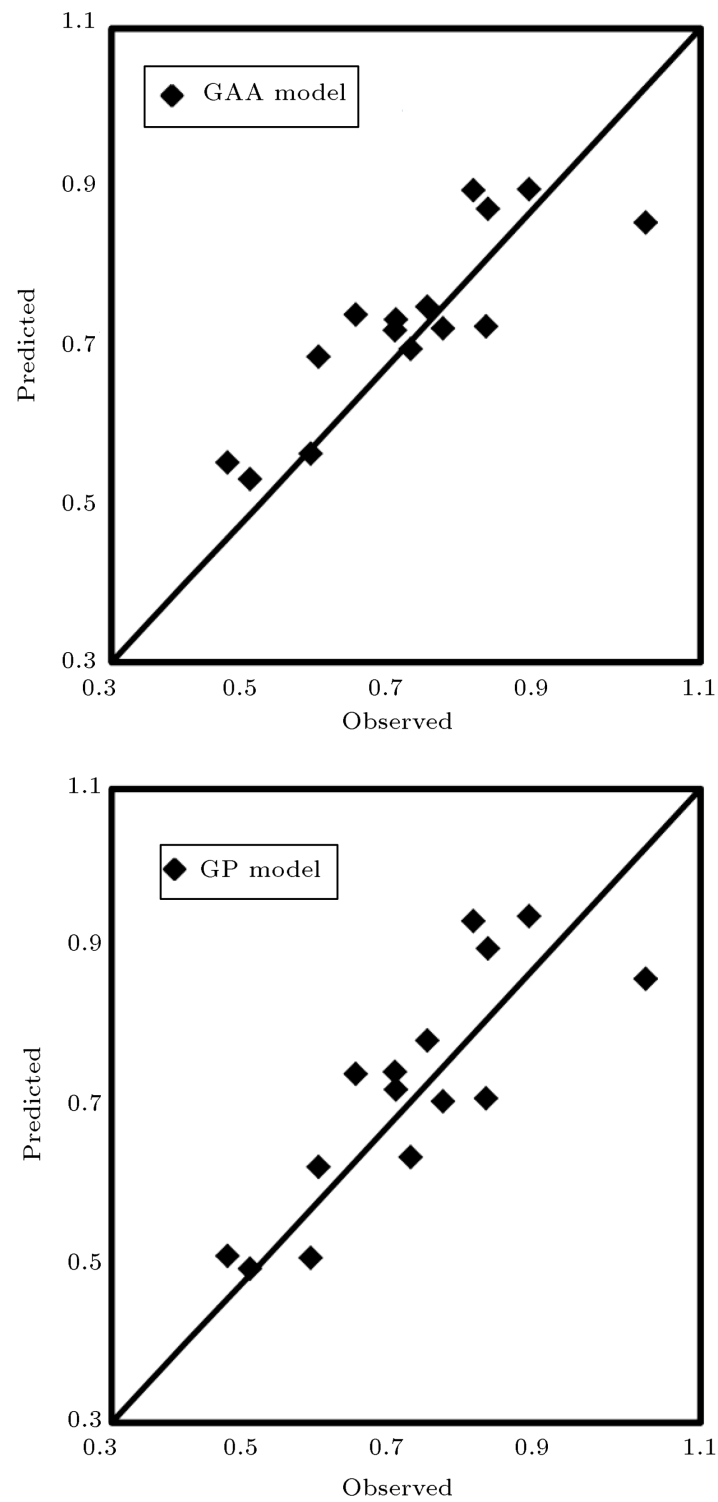

Figure 3. Comparison of the GAA and GP mean bed shear stress estimates with the observations in the test status.

however, in the equation presented by Knight [2], the aspect ratio is the only effective parameter. In Figure 4, the GAA model data overestimate the value of the mean bed shear stress for $\frac{\bar{\tau}_{b}}{\rho g h S}>0.73$ and underestimate the value in $\frac{\bar{\tau}_{b}}{\rho g h S}<0.73$. In addition, in the GAA model, fewer data are in the range of the $\pm 10 \%$ error. Equation of Knight [2] underestimates the mean bed shear stress value more than the GAA model does. When the underestimated values predict bed shear stress, the erosion occurs in the channel since the actual values of shear stress are higher than the predicted values, and the designed channel is poor in erosion. Therefore, the equation is used which is obtained by the GAA due to designing more stable channels.

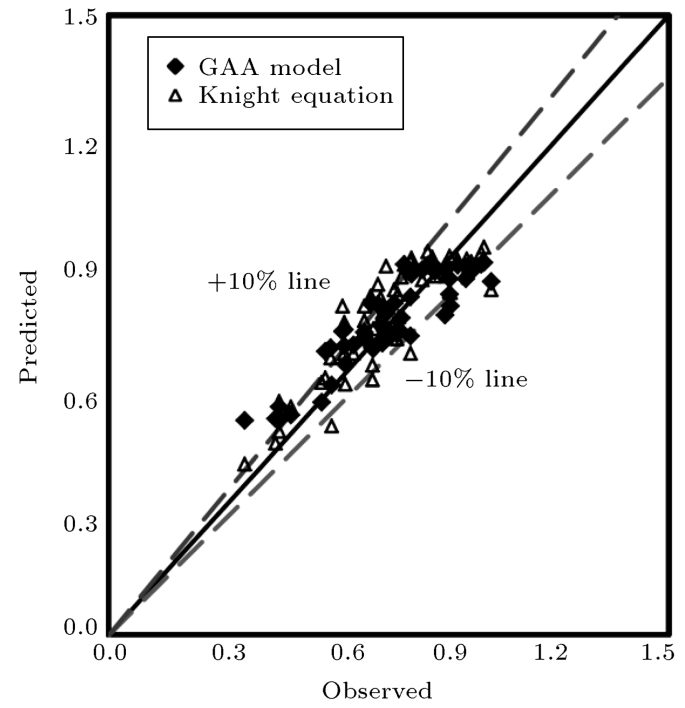

Figure 4. Comparison of the performance of the GAA model with the equation of Knight [2].

\section{Conclusions}

The distribution of the bed shear stress is effective in the shape of the bed topography channels in sediment transportation. In this study, the GAA and GP models, as two methods of the GA, were compared to predict the mean bed shear stress in a rectangular channel with non-homogeneous roughness. Both soft computing models were extended and the performance of each model in each step was evaluated. For each model, several input combinations and fitness functions were studied to recognize the best state among them. The statistical parameters were used to evaluate predicted mean bed shear stress values of the GAA and GP models. The results showed the best program for the GP model obtained by input combination (ii), MSE fitness function, and $(+,-, \times, \div)$ mathematical functions. The best formula in the GAA model was concluded with input combination (ii), MSE fitness function, and log-pur transfer function. The accuracy of the GAA and GP techniques in predicting this object was investigated, and the GAA model was introduced as a more powerful model compared with GP model.

The results showed that the GP model has the ability to estimate the mean bed shear stress, but the GAA model has smaller values of statistical parameters $(\mathrm{RMSE}=0.0731, \mathrm{MAE}=0.0607$, and $\% \delta=8.0451)$, and hence performs better than the GP model. Finally, the obtained equation with the GAA model was compared with that presented by Knight [2]. In addition to being simple, this equation was better in estimating the mean bed shear stress than that of Knight [2]. The obtained equation by the GAA method can be used instead of the equation proposed by Knight [2] in calculating mean bed shear stress. This study suggests that the proposed GAA model is strong and useful in 
predicting the mean bed shear stress in a rectangular channel with rough boundaries.

\section{References}

1. Knight, D.W., Demetriou, J.D. and Hamed, M.E. "Boundary shear in smooth rectangular channels", $J$. Hydraul. Eng., 10, pp. 405-422 (1984).

2. Knight, D.W. "Boundary shear in smooth and rough channels", J. Hydraul. Div., 107, pp. 839-851 (1981).

3. Tominaga, A., Nezu, I., Ezaki, K. and Nakagawa, H. "Three-dimensional turbulent structure in straight open channel flows", J. Hydraul. Res., 27, pp. 149-173 (1989).

4. Bonakdari, H., Tooshmalani, M. and Sheikh, Z. "Predicting shear stress distribution in rectangular channels using entropy concept", Int. J. Eng., 28(3), pp. 357-364 (2015).

5. Alhamid, A.A.I. "Boundary shear stress and velocity distributions in differentially roughened trapezoidal open channels", PhD Thesis, The University of Birmingham (1991).

6. Sheikh, Z. and Bonakdari, H. "Prediction of boundary shear stress in circular and trapezoidal channels with Entropy concept", Urban Water J., 13(6), pp. 629-636 (2015).

7. Knight, D.W., Yuen, K.W.H. and Al Hamid, A.A.I. "Boundary shear stress distributions in open channel flow", In: K. Beven, P. Chatwin, J. Millbank (Eds.), Physical Mechanisms of Mixing and Transport in the Environment, Wiley New York, pp. 51-87 (1994).

8. Ardiclioglu, M., Sekcin, G. and Yurtal, R. "Shear stress distributions along the cross section in smooth and rough open channel flows", Kuwait J. Sci. Eng., 33, pp. 155-68 (2006).

9. Guo, J. and Julien, P.Y. "Shear stress in smooth rectangular open-channel flow", J. Hydraul. Eng., 131(1), pp. 30-37 (2005).

10. Babaeyan-Koopaei, K., Ervine, D.A., Carling, P.A. and Cao, Z. "Velocity and turbulence measurements for two overbank flow events in River Severn", $J$. Hydraul. Eng., 128, pp. 891-900 (2002).

11. Julien, P.Y., Erosion and Sedimentation, U.K. Cambridge University Press (1995).

12. Berlamont, J.E., Trouw, K. and Luyckx, G. "Shear stress distribution in partially filled pipes", J. Hydraul. Eng., 129(9), pp. 697-705 (2003).

13. Han, Y., Yang, S.Q. and Dharmasiri, N. "Application of main flow data in the determination of boundary shear stress in smooth closed ducts", World Environmental and Water Resources Congress: Crossing Boundaries, Albuquerque, NM (2012).

14. Yang, S.Q., Dharmasiri, N. and Han, Y. "Momentum balance method and estimation of boundary shear stress distribution", J. Hydraul. Eng., 138, pp. 657660 (2012).
15. Knight, D.W. and Sterling, M. "Boundary shear in circular pipes partially full", J. Hydraul. Eng., 126(4), pp. 263-275 (2000).

16. Bonakdari, H., Sheikh, Z. and Tooshmalani, M. "Comparison between Shannon and Tsallis entropies for prediction of shear stress distribution in circular open channels", Stoch. Env. Res. Risk Assess., 29(1), pp. 1-11 (2015).

17. Sheikh Khozani, Z., Bonakdari, H. and Ebtehaj, I. "An analysis of shear stress distribution in circular channels with sediment deposition based on gene expression programming", Int. J. Sediment Res. (2017). DOI: org/10.1016/j.ijsrc.2017.04.004 (2017).

18. Gharagheizi, F., Ilani-Kashkouli, P., Farahani, N. and Mohammadi, A.H. "Gene expression programming strategy for estimation of flash point temperature of non-electrolyte organic compounds", Fluid Phase. Equilibria., 329, pp. 71-77 (2012).

19. Sheikh Khozani, Z., Bonakdari, H. and Zaji, A.H. "Estimating the shear stress distribution in circular channels based on the randomized neural networks technique", Appl. Soft Comp., 58, pp. 144-148 (2017).

20. Najafzadeh, M., Barani, G.A. and Hessami Kermani, M.R. "GMDH based back propagation algorithm to predict abutment scour in cohesive soils", Ocian Eng., 59, pp. 100-106 (2013).

21. Kisi, O., Emin Emiroglu, M.E., Bilhan, O. and Guven, A. "Prediction of lateral outflow over triangular labyrinth side weirs under subcritical conditions using soft computing approaches", Expert Syst. Appl., 39, pp. 3454-3460 (2012).

22. Shiri, J., Ashraf Sadraddini, A., Nazemi, A.H., Kisi, O., Landeras, G., Fakheri Fard, A. and Marti, P. "Generalizability of gene expression programmingbased approaches for estimating daily reference evapotranspiration in coastal stations of Iran", J. Hydrol., 508, pp. 1-11 (2014).

23. Sheikh Khozani, Z., Bonakdari, H. and Zaji, A.H. "Using two soft computing methods in prediction wall and bed shear stress in smooth rectangular channels", Appl. Water Sci. (2017). DOI: 10.1007/s13201-0170548-y (2017).

24. Azamathulla, H.M. and Zahiri, A. "Flow discharge prediction in compound channels using linear genetic programming", J. Hydrol., 454-455, pp. 203-207 (2012).

25. Zaji, A.H. and Bonakdari, H. "Performance evaluation of two different neural network and particle swarm optimization methods for prediction of discharge capacity of modified triangular side weirs", Flow Meas. Instrum., 40, pp. 149-156 (2014).

26. Tayfur, G. "Artificial neural networks for sheet sediment transport", Hydrol. Sci. J., 47, pp. 879-892 (2002).

27. Kisi, O., Dailr, A.H., Cimen, M. and Shiri, J. "Suspended sediment modeling using genetic programming and soft computing techniques", J. Hydrol., 450-451, pp. $48-58$ (2012). 
28. Cobaner, M., Seckin, G., Seckin, N. and Yurtal, R. "Boundary shear stress analysis in smooth rectangular channels and ducts using neural networks", Water Environ. J., 24, pp. 133-139 (2010).

29. Sheikh Khozani, Z., Bonakdari, H. and Zaji, A.M. "Application of soft computing technique in prediction percentage of shear force carried by walls in rectangular channel with non-homogenous roughness", Water Sci. Technol., 73(1), pp. 124-129 (2016).

30. Sheikh Khozani, Z., Bonakdari, H. and Zaji, A.M. "Application of a genetic algorithm in predicting the percentage of shear force carried by walls in smooth rectangular channels", Measurement, 87, pp. 87-98 (2016).

31. Zadeh, M.R., Amin, S., Khalili, D. and Singh, V.P. "Daily outflow prediction by multi layer perceptron with logistic sigmoid and tangent sigmoid activation functions", Water Resour. Manage., 24, pp. 2673-2688 (2010).

32. Emiroglu, M.E., Bilhan, O. and Kisi, O. "Neural networks for estimation of discharge capacity of triangular labyrinth side-weir located on a straight channel", Expert Sys. Appl., 38, pp. 867-874 (2011).

33. Pierini, J.O., Gomez, E.A. and Telesca, L. "Prediction of water flows in Colorado River, Argentina. Lat Am", J. Aquatic. Res., 40, pp. $872-880$ (2012).

34. Levenberg, K. "A method for the solution of certain non-linear problems in Least-Squares", Qu. Appl. Math., 2(2), pp. 164-168 (1944).

35. Koza, J.R. "Genetic programming as a means for programming computers by natural selection", Stat. Comput., 4, pp. 87-112 (1994).

\section{Biographies}

Zohreh Sheikh Khozani is a PhD student in Hydraulic structures (Civil Engineering), Faculty of Engineering, University of Razi, Kermanshah, Iran. She obtained MSc degree at Semnan University in 2011.
She has published 16 papers in ISI journals and more than 8 conference presentations. She works in the field of shear stress distribution and sediment transport in open channels and rivers and use of entropy concept and soft computing methods in engineering applications.

Hossein Bonakdari is a Professor at the Department of Civil Engineering of University of Razi. He earned his $\mathrm{PhD}$ degree in Civil Engineering at the University of Caen-France. Dr. Bonakdari had joined University of Razi as a faculty member in 2006; He has supervised $4 \mathrm{PhD}$ and $25 \mathrm{MS}$ theses. He has teaching experience of more than 15 years in the field of Civil Engineering. Furthermore, From 2013 till 2015, he was the Director General of Training, Research and Technology Development at Ministry of Energy, Iran, and also from 2011-2013, he was the Deputy of Planning \& Development in National Water and Wastewater Engineering Company, Iran. His fields of specialization and interest include: practical application of soft computing in engineering, modeling of wastewater urban drainage systems, sediment transport, computational fluid dynamic and hydraulics, design of hydraulic structures, and fluid mechanics. From 2010 till 2011, he has been a researcher at Laboratory of Civil and Environmental Engineering, INSA of Lyon, France. Results obtained from his studies have been published in more than 100 papers in international journals (h-index $=12$ ). He has also more than 150 presentations in national and international conference. He has published two books, and has been rated as a distinguished researcher in the Razi University from 2014 to 2015.

Amir Hossein Zaji is a PhD student in Hydraulic structures, Department of Civil Engineering, University of Razi, Kermanshah, Iran. He has 20 published papers in ISI journals. He works in the field of soft computing methods in engineering applications. 